\title{
PDF fit in the fixed-flavor-number scheme
}

\author{
S. Alekhin ${ }^{a, b}$, J. Blümlein ${ }^{a,}$, and S. Moch ${ }^{a}$, \\ ${ }^{a}$ Deutsches Elektronensynchrotron DESY \\ Platanenallee 6, D-15735 Zeuthen, Germany \\ ${ }^{b}$ Institute for High Energy Physics \\ 142281 Protvino, Moscow region, Russia
}

\begin{abstract}
We discuss the heavy-quark contribution to deep-inelastic scattering in the scheme with $n_{f}=3,4,5$ fixed flavors. Based on the recent ABM11 PDF analysis of world data for deep-inelastic scattering and fixed-target data for the Drell-Yan process with the running-mass definition for heavy quarks, we show that the fixed flavor number scheme is sufficient for describing the deep-inelastic-scattering data in the entire kinematic range. We compare with other PDF sets and comment on the implications for measuring the strong coupling constant $\alpha_{s}\left(M_{Z}\right)$.
\end{abstract}

Keywords: parton distributions, heavy quarks, strong coupling constant

Account of the heavy-quark contribution to deepinelastic scattering (DIS) is an important issue for the PDF fits. The charm- and bottom-quark production, which is mostly relevant for the existing data analysis, is substantial at small values of the Bjorken variable $x$ forming an important contribution to the inclusive structure functions (SFs) measured at HERA. Furthermore, the semi-inclusive SFs, which correspond to the subprocess with the heavy quarks in the final state, can provide an additional constraint on the small- $x$ gluon distribution [1]. However, the calculation of the heavy-quark production cross section is hampered within perturbative QCD because the higher-order corrections are extremely involved for the case of two scales, the lepton momentum transfer $Q^{2}$ and the heavy-quark mass. At present the QCD corrections to the massive Wilson coefficients are known up to NLO only $[2,3,4]$. The partial NNLO corrections stemming from the soft-gluon threshold re-summation have been also calculated for the neutral-current (NC) [5] and charged-current (CC) heavy-quark production [6]. For the case of electroproduction they are numerically important at small $x$ and $Q^{2}$ reaching $\mathrm{O}(10 \%)$ in the kinematic region of HERA. The NNLO threshold re-summation calcula- tions of Ref. [5] were recently updated [7, 8] and now they include all threshold-enhanced logs and the Coulomb term. The heavy-quark mass appearing in the massive Wilson coefficients of Refs. [1, 2, 4, 8] corresponds to the pole mass which emerges in the QCD Lagrangian. The pole-mass definition provides a straightforward way for the perturbative calculations, however, it is not ideal for phenomenology since the pole mass is quite sensitive to the QCD radiative corrections. This shortcoming is eliminated when the heavyquark mass is defined in the $\overline{\mathrm{MS}}$-scheme, similarly to the strong coupling constant $\alpha_{s}$. The heavy quark $\overline{\mathrm{MS}}$ masses are conventionally parametrized at the scales of the heavy-quark mass itself. These scales are close to the typical DIS hard-scattering scale therefore their perturbative stability is greatly enhanced if compared to the pole-mass definition. The massive Wilson coefficients re-calculated in terms of the running mass also demonstrate improved perturbative stability and the reduced renormalization/factorization scale sensitivity at the HERA kinematics [9]. In this range only the charmand bottom-quark production are relevant. The $c$ - and $b$-quark $\overline{\mathrm{MS}}$-masses averaged over different determina- 

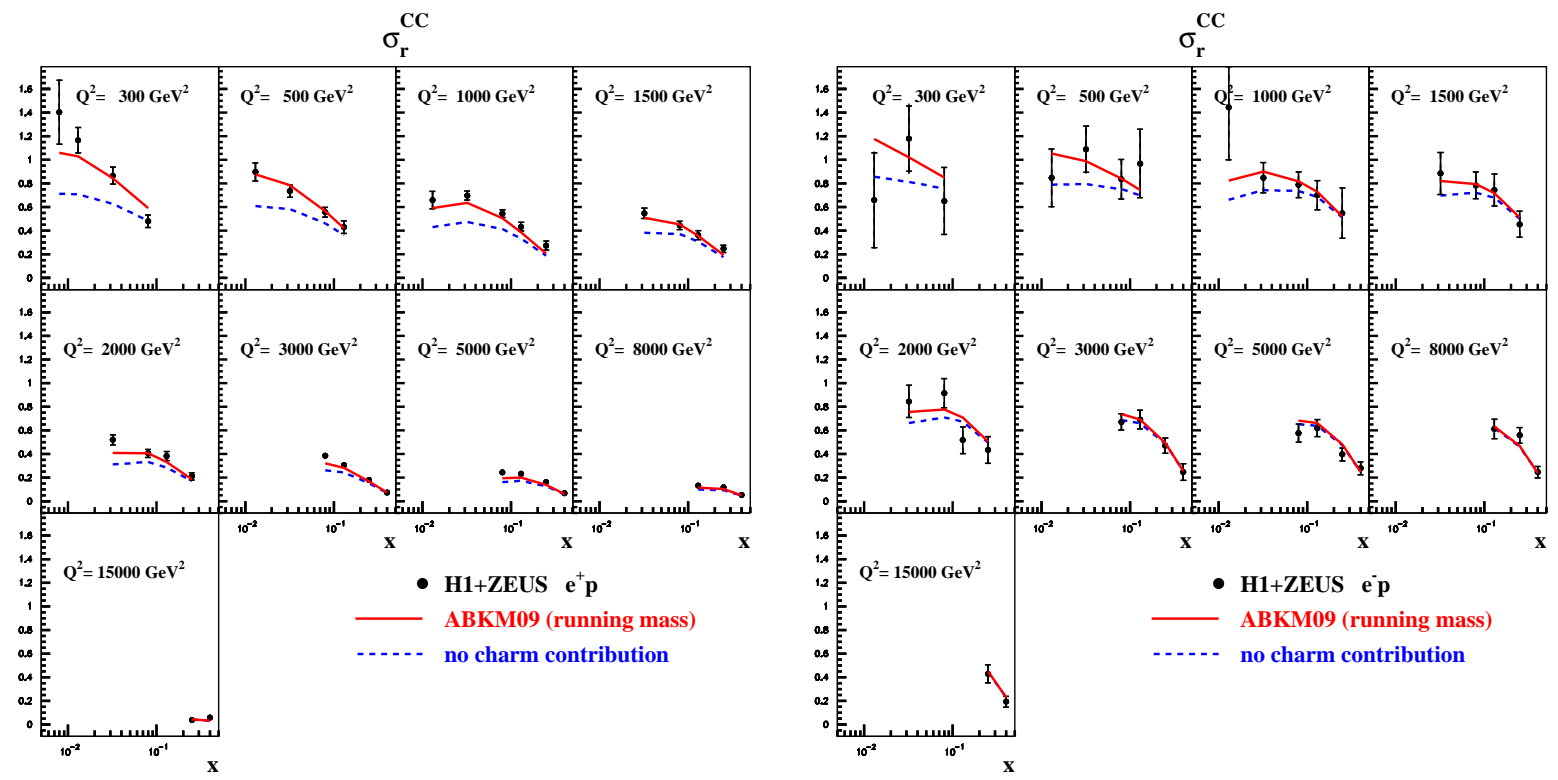

Figure 1: The CC inclusive cross section data obtained at HERA [10] with the positron beam (left) and the electron beam (right) compared with the running-mass 3-flavor scheme predictions based on the NNLO PDFs of Ref. [9] (solid lines). The same predictions without the $c$-quark contribution taken into account are given for comparison (dashes).

tions are

$$
m_{c}\left(m_{c}\right)=1.27 \pm 0.08 \mathrm{GeV}
$$

and

$$
m_{b}\left(m_{b}\right)=4.19 \pm 0.13 \mathrm{GeV}
$$

respectively [11]. The uncertainties in these values are essentially smaller than the spread in the heavy-quark masses values employed in the various global PDF fits, cf. Tab. 1 in [9]. Therefore the running-mass definition provides a footing for the consolidation of those PDFs.

Taking advantage of the running-mass definition and new massive NNLO corrections we update our ABKM09 PDFs, which were obtained within the fixedflavor-number (FFN) scheme at NLO and NNLO [12], and produce in this way a new PDF set, ABM11. Apart from this theoretical improvement we add to the fit recently published HERA data. Firstly, the separate H1 [13] and ZEUS [14] data sets on the NC inclusive SFs are replaced by much more accurate combined HERA data [10]. The unprecedentedly small uncertainty of $1-2 \%$, including the normalization error of $0.5 \%$, achieved for the combined HERA inclusive data allows to reach better constraints on the small- $x$ PDFs as compared to the ABKM09 analysis. Only the NC combined HERA data with $Q^{2}<1000 \mathrm{GeV}^{2}$ are used in order to exclude contributions due to the $Z$-boson exchange without loosing the statistical significance of the NC HERA data for our fit. Besides, we include into the fit the CC HERA data obtained for the electron and positron beams [10]. The CC HERA sample ranges up to $Q^{2}=15000 \mathrm{GeV}^{2}$. To demonstrate that the treatment of the charm production within the FFN scheme is applicable at that large transverse momentum we compared the predictions based on the variant of the NNLO ABKM09 fit performed with the running-mass scheme [9]. The value of $m_{c}\left(m_{c}\right)=1.18 \pm 0.06 \mathrm{GeV}$ is obtained in this fit with the constraint of Eq. (1) imposed. Taking this value of $m_{c}\left(m_{c}\right)$ we find very good agreement with the CC HERA data in the whole range of $Q^{2}$, cf. Fig. 1. At small $x$ the charm contribution to the inclusive $\mathrm{CC}$ cross sections is quite significant, similarly to the case of the NC DIS. Since the CC charm production is initiated by the strange quarks mainly, this sample provides an additional constraint on the small- $x$ strange sea distribution, which is at the moment defined by the neutrino-nucleon DIS data only [15]. In the same way the charged-lepton initiated CC data can be used to determine the value of the $c$-quark mass, in particular employing the potential of the planned high-luminosity EIC facility [16]. Finally, we add to the fit the inclusive H1 data obtained in a special HERA run with high inelasticity $y$ achieved [17]. At the kinematics probed in this run the inclusive SFs are sensitive to the structure 
function $F_{L}$ at small $x$ and $Q^{2}$ and thereby shed light on the details of the small- $x$ QCD dynamics, in particular the shape of the gluon distribution at small $x$.

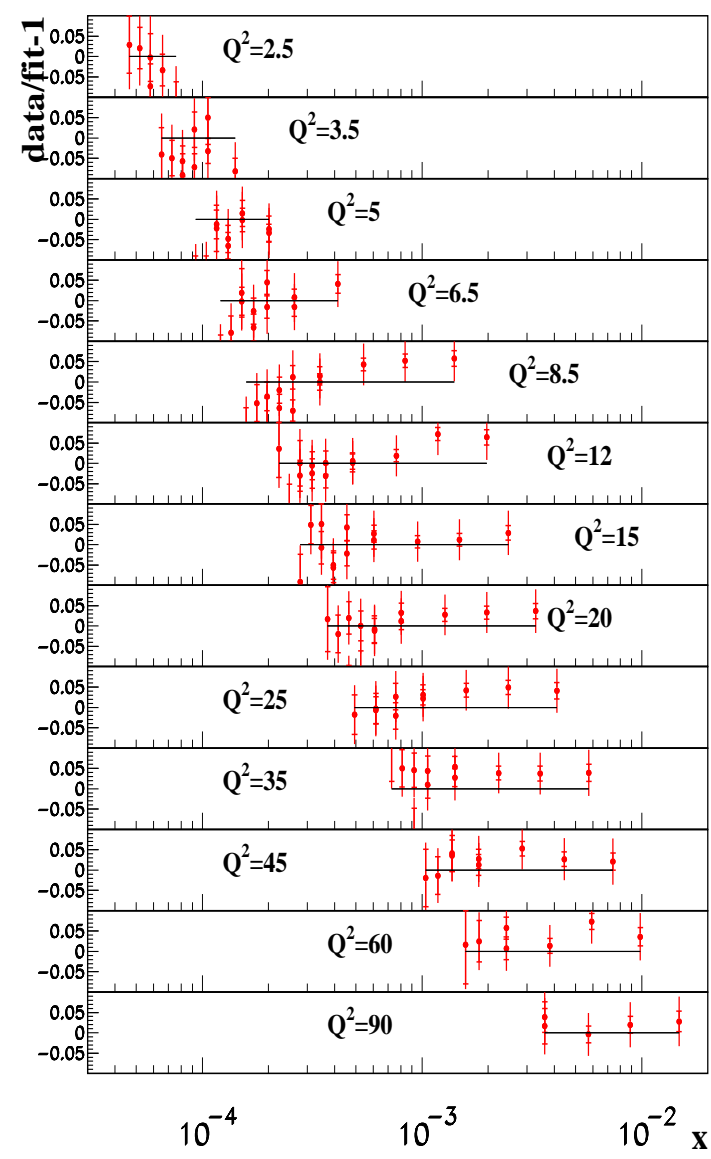

Figure 2: The pulls versus $x$ for the H1 NC inclusive DIS c.s. data of Ref. [17] binned on the momentum transfer $Q^{2}$ in units of $\mathrm{GeV}^{2}$ w.r.t. the ABM11 NNLO fit. The data points with different inelasticity $y$ still may overlap in the plot. The inner bars show statistical errors in data and the outer bars the statistical and systematical errors combined in quadrature [18].

Similar to the ABKM09 analysis, in ABM11 the HERA data are supplemented by the ones on dimuon production in the (anti)neutrino-nucleon DIS and the Drell-Yan process in order to separate the quark PDFs by flavors. Also we employ the fixed-target inclusive DIS data obtained in the NMC [25], the BCDMS [26, 27], and the SLAC experiments [28, 29, 30, 31, 32, 33]. The fixed-target data allow to constrain the PDFs at large $x$. However, modeling of the higher-twist terms is required since they contribute to the DIS SFs at small $Q^{2}$ [34]. The corrections for nuclear effects [35] are also taken into account in the analysis of fixed target

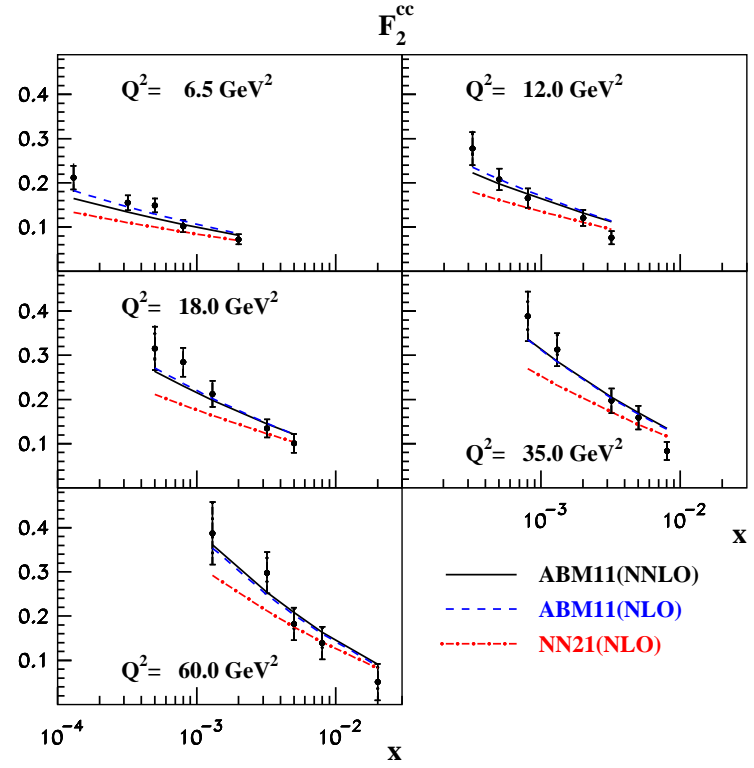

Figure 3: The predictions for the semi-inclusive structure function $F_{2}^{c c}$ at different values of the momentum transfer $Q^{2}$ versus $x$ for the ABM11 PDFs in NNLO (solid curves), the ABM11 PDFs in NLO (dashes), and the NN21 PDFs [19] in NLO (dashed dots), all taken in the 3-flavor running-mass scheme with the the value of $m_{c}=1.27 \mathrm{GeV}$ [11]. The NNLO predictions based on the MSTW [20] and JR09 [21] PDFs (not displayed in the plot) strongly overlap with the ABM11 ones. The H1 data of Ref. [22] given in the plot are extracted with the HVQDIS code of Ref. [23] .

data when relevant, cf. [18] for details. The overall quality of the fit is quite good. For 3036 data points used in the fit the value of $\chi^{2}$ is 3391 and 3378 for the NLO and NNLO variants, respectively. For the combined HERA data [10] including the NC and CC samples the value of $\chi^{2} / N D P=537 / 486$ is obtained at NNLO with full account of the systematics error correlations. The pulls of NC HERA data w.r.t. the fit do not demonstrate a statistically significant trend versus $Q^{2}$. This gives additional justification for application of the FFN scheme to the analysis of the DIS data at realistic kinematics, cf. also [36]. At small $x$ and $Q^{2}$ the perturbative QCD corrections rise and they are particularly big for the NNLO corrections to the massless Wilson coefficients for $F_{L}$ [37]. With account of these corrections the high- $y \mathrm{H} 1$ data [17], which are quite sensitive to the contribution from $F_{L}$ are described pretty well, with the value of $\chi^{2}=137$ for 130 data points. Also the H1 data do not suggest a violation of the conventional QCD evolution down to $x \sim 10^{-5}$, cf. Fig. 2. In this way we do not confirm a hint on the evolution kernel re- 


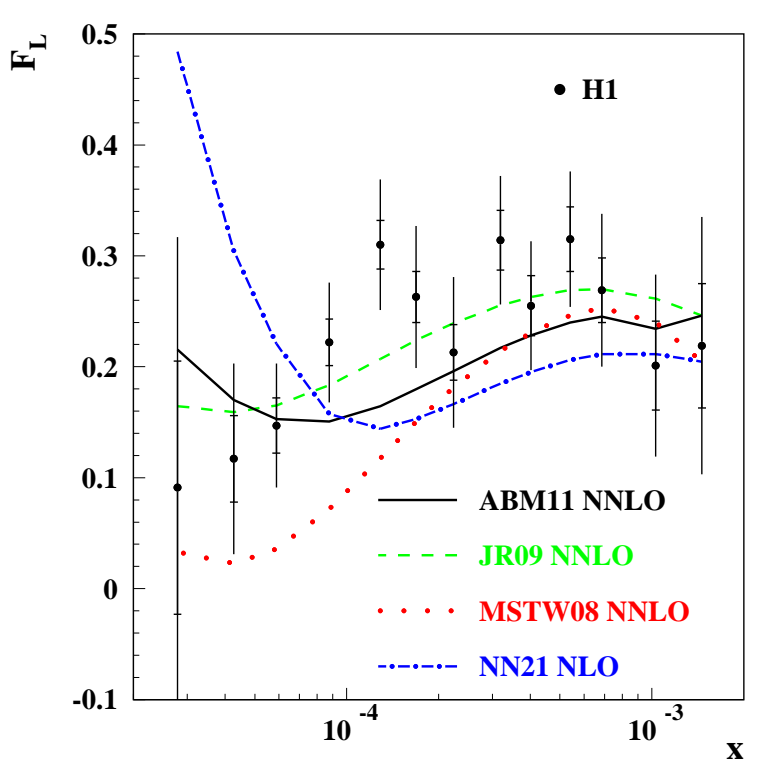

Figure 4: The data on $F_{L}$ versus $x$ obtained by the H1 collaboration [17] confronted with the 3-flavor scheme NNLO predictions based on the different PDFs (solid line: this analysis, dashes: JR09 [21], dots: MSTW08 [20]). The NLO predictions based on the 3-flavor NN21 PDFs [19] are given for comparison (dashed dots). The value of $Q^{2}$ for the data points and the curves in the plot rises with $x$ in the range of $1.5 \div 45 \mathrm{GeV}^{2}[18]$.

summation effects observed in the NNPDF fit [38]. It is worth noting in this connection that the interpretation of the low- $x$ DIS inclusive data is sensitive to the treatment of the heavy-quark contribution. While we employ in the analysis of DIS data the FFN scheme with 3 flavors, in many other PDF fits [20, 24, 39] the variable-flavornumber (VFN) scheme is used. The VFN approach to the description of the DIS is based on the asymptotic expressions for massive Wilson coefficients taken in the 3-flavor FFN scheme [40, 41], which are valid at $Q^{2} \gg m_{h}^{2}$, where $m_{h}$ is the heavy-quark mass. In this limit the power corrections vanish and the structure functions come down to convolutions of the massless Wilson coefficients with the PDFs, which now are defined in the 4-flavor scheme including also the heavyquark PDFs. The matching conditions between 3- and 4-flavor schemes are known up to the second order in $\alpha_{s}[40,41]$ and the first third-order corrections are also available [42, 43]. However, in order to employ the VFN scheme in the analysis of realistic data, which range down to $Q^{2} \sim m_{h}^{2}$, one has to take into account the power corrections as well. This task is conceptually difficult within the VFN formalism therefore the power

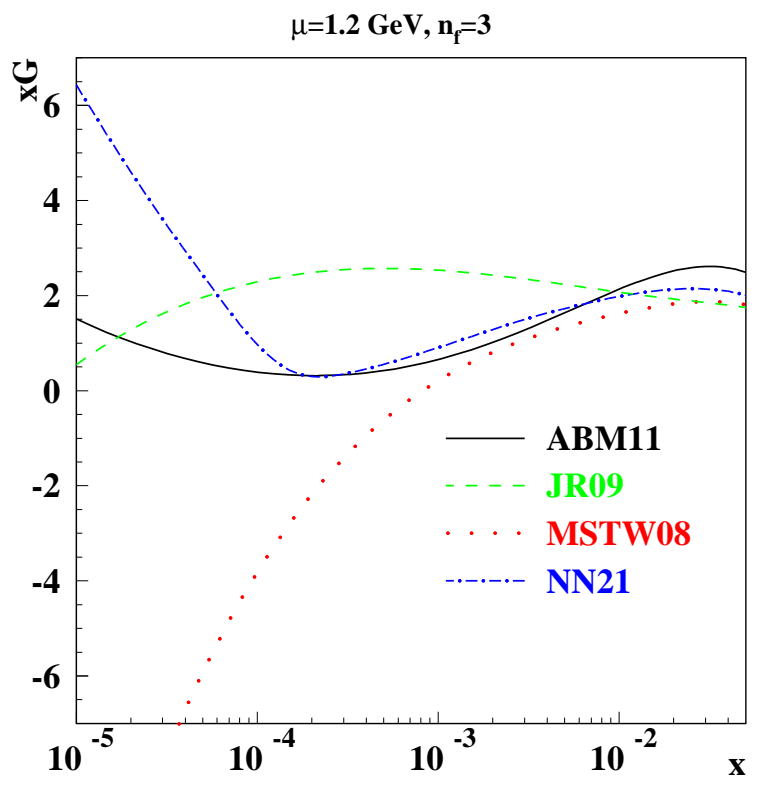

Figure 5: The 3-flavor gluon distributions obtained in the various NNLO fits at the scale of $\mu=1.2 \mathrm{GeV}$ (solid line: this analysis, dashes: JR09 [21], dots: MSTW08 [20], dashed dots: NN21 [24]).

corrections are commonly modeled using the combination of the massive FFN and massless VFN Wilson coefficients [44]. The models which are obtained in this way pretend to describe the full-range kinematics of existing DIS data; therefore they should reproduce the 3-flavor scheme results at small $Q^{2}$. A particular shape of the massive VFN coefficient functions at $Q^{2} \ngtr m_{h}^{2}$ is subject to a particular choice and there are numerous VFN scheme prescriptions, which differ basically by the degree of smoothness provided for matching with the FFN scheme. In cases the smoothness is achieved by introducing empirical parameter(s), which control dumping of the massless VFN term at small $Q^{2}$ [45]. It is worth noting that the uncertainty in a particular choice of these parameters is propagated into the uncertainties in PDFs obtained with such prescriptions. Moreover, additional parameters, which appear in the coefficient functions of Ref. [45] do not enter into the QCD anomalous dimensions. Therefore the QCD factorization may be broken in this way. In contrast, the NNLO S-ACOT- $\chi$ prescription [46] is explicitly based on the factorization theorem and does not contain damping factors. However the matching smoothness is not guaranteed in this case and the S-ACOT- $\chi$ based calculations overshoot the FFN scheme results at $Q^{2} \sim m_{h}^{2}$, cf. Fig. 4 in [46]. On the other hand, the BMSN prescription [40] ensures smooth 


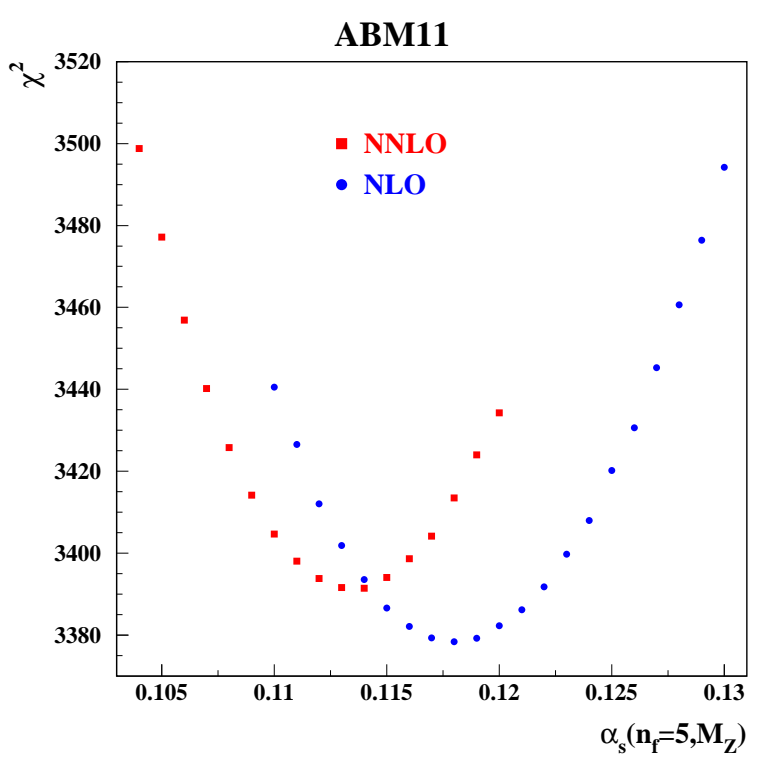

Figure 6: The $\chi^{2}$-profile as a function of $\alpha_{s}\left(M_{Z}\right)$ in the present analysis at NLO (circles) and NNLO (squares) [18].

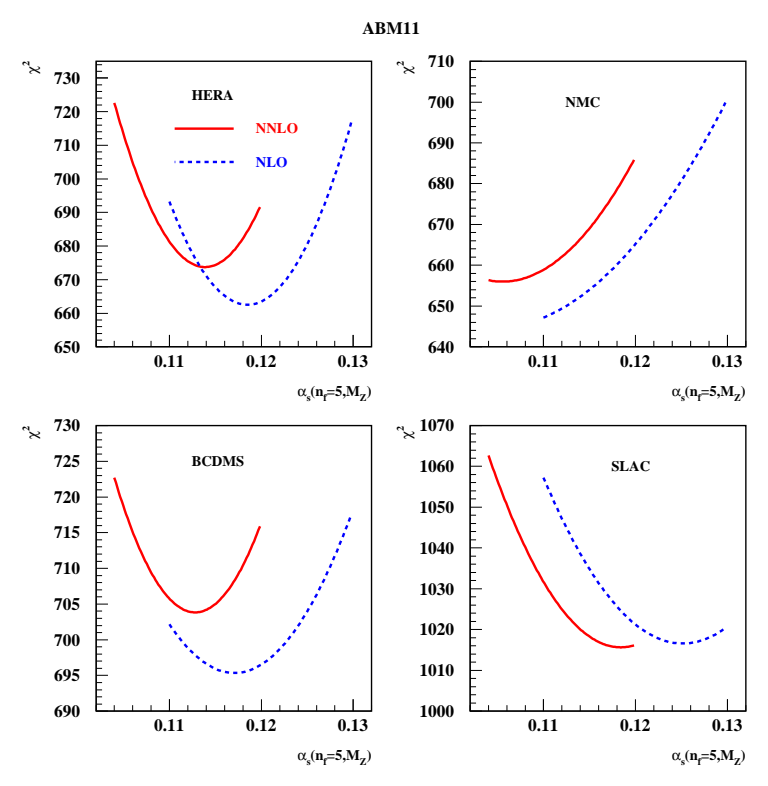

Figure 7: The $\chi^{2}$-profile versus the value of $\alpha_{s}\left(M_{Z}\right)$, for the HERA data [10, 17], the NMC data [25], the BCDMS data [26, 27], and the SLAC data [28, 29, 30, 31, 32, 33], all obtained in variants of the present analysis with the value of $\alpha_{s}$ fixed and all other parameters fitted (solid lines: NNLO fit, dashes: NLO one) [18].

matching with the FFN scheme at $Q^{2}=m_{h}^{2}$ inherently, without additional parameters. Furthermore the results of the PDF-analysis are not sensitive to the choice of ei- ther the VFN scheme as the BMSN prescription or the FFN scheme [12].

To a certain extent different VFN scheme prescriptions can be sorted out with the data on the semiinclusive structure function $F_{2}^{c c}$, which correspond to the DIS sub-process with two charmed quarks in the final state. Strictly speaking this observable is infrared unsafe due to the non-singlet term contributing to perturbative QCD calculations starting from NLO. Rigorous infrared safety of $F_{2}^{c c}$ can be restored only with an additional soft cut, e.g. imposed on the $c \bar{c}$ invariant mass [47]. At the same time the non-singlet contribution in $F_{2}^{c c}$ is numerically small and it can be safely disregarded in the analysis of the existing DIS data. The recent $\mathrm{H} 1$ data on $F_{2}^{c c}$ [22] are compared to the predictions based on different PDFs in Fig. 3. To provide a comparison consistently we employ the FFN scheme with the running-mass definition, taking the corresponding 3-flavor PDFs provided by different groups and the world-average value of $m_{c}\left(m_{c}\right)=1.27 \mathrm{GeV}$ [11]. This value was also used in our analysis, however, the data of Ref. [22] are not included into our fit. The calculations are performed with the open-source code OPENQCDRAD $^{1}$, which contains the implementation of the DIS Wilson coefficients up to NNLO. The NNLO ABM11 predictions are in a good agreement with the data. Also they agree with the combined $\mathrm{H} 1$ and ZEUS data on $F_{2}^{c c}$, which range wider in kinematics [48]. The NLO ABM11 calculations are very close to the NNLO ones that reflect the improved perturbative stability of the running-mass definition scheme. The NNLO predictions based on the MSTW [20] and JR09 [21] PDFs are in a good agreement with ours and with the $\mathrm{H} 1$ data, while the NLO NN21 [19] predictions systematically undershoot the data. The NN21 PDFs are obtained with the FONLL prescription of the VFN scheme [49]. The FONLL variant of the VFN scheme is conceptually similar to the prescription of Ref. [45], however, it allows less flexible modeling of the massive coefficient functions at $Q^{2} \ngtr m_{h}^{2}$. In any case the VFN modeling should reproduce the FFN scheme results at small $Q^{2}$. However, the trend demonstrated by the $\mathrm{NN} 21$ predictions is opposite and they also diverge from the $\mathrm{H} 1$ data at small $Q^{2}$.

The high-y H1 data [17] also discriminate different PDFs. The predictions for $F_{L}$ at low $x$ computed with various NLO and NNLO PDFs are compared with the $\mathrm{H} 1$ data in Fig. 4. As for the comparison with $F_{2}^{c c}$ above, we employ the FFN scheme with running-mass

\footnotetext{
${ }^{1}$ http://www-zeuthen.desy.de/ ${ }^{\sim}$ alekhin/OPENQCDRAD
} 

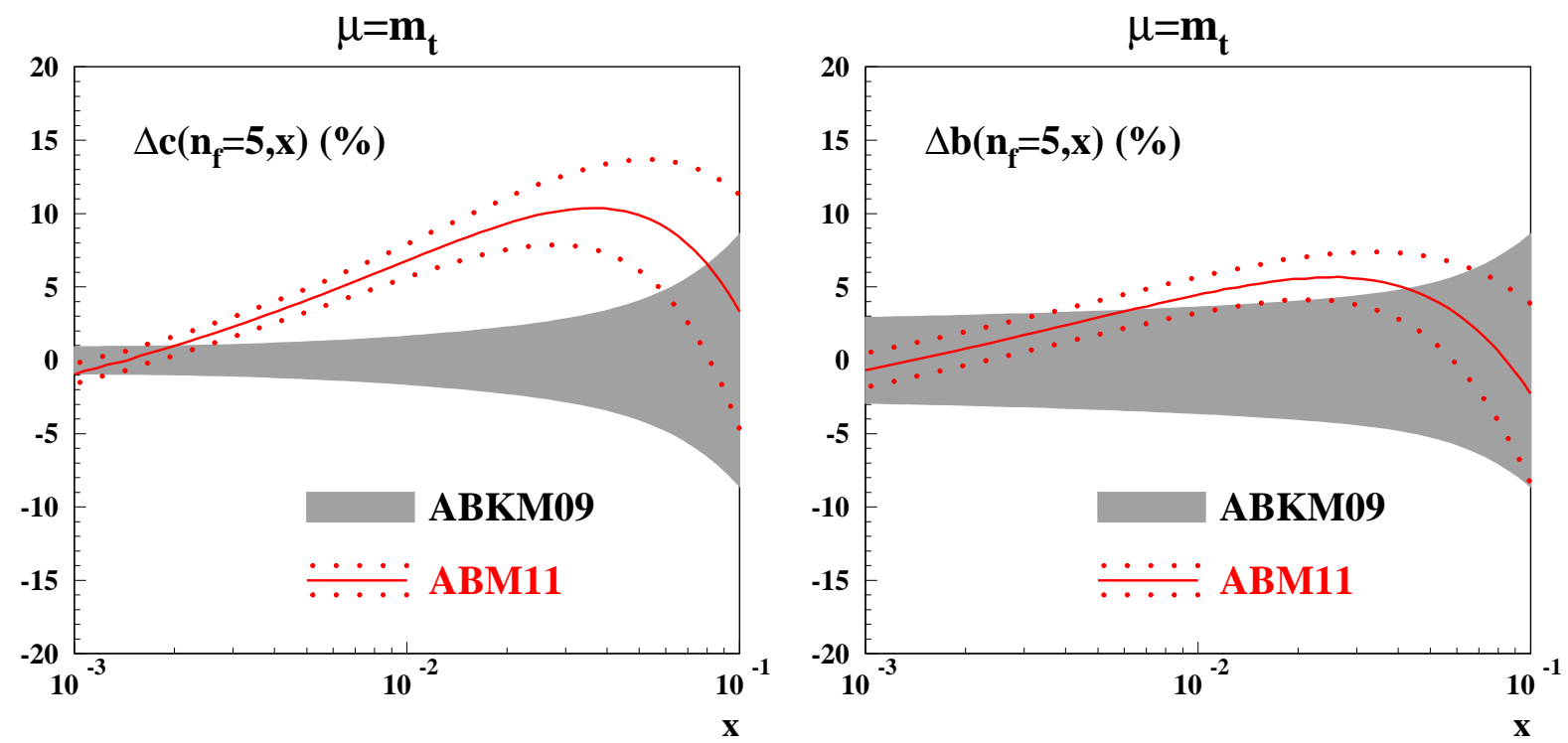

Figure 8: The charm- (left) and the bottom-quark (right) PDFs obtained in the global fit: The dotted (red) lines denote the $\pm 1 \sigma$ band of relative uncertainties (in percent) and the solid (red) line indicates the central prediction resulting from the fit with the running masses [9]. For comparison the shaded (gray) area represents the results of ABKM09 [12] [18].

definition and the publicly available 3-flavor PDFs. The NNLO ABM11 and JR09 predictions obtained in this way demonstrate good agreement with the data in the whole range of $x$, while the MSTW predictions undershoot the data at low $x$. This is correlated with the strong fall-off of the MSTW gluon distributions at small $x$ taking negative values at $x \lesssim 10^{-3}$ at small scales, $\mathrm{cf}$. Fig. 5. The observed discrepancy cannot be attributed to the impact of a particular choice of the VFN scheme prescription in the MSTW fit since the low- $x$ tail of the H1 data correspond to small $Q^{2}$, where the VFN scheme employed in the MSTW fit reproduces the FFN one. Due to this the small- $x$ MSTW gluon distribution should move up and consolidate with the JR09 and ABM ones once the H1 data [17] are included into the MSTW fit. The same is also valid for the NN21 PDFs, which overshoot the H1 data at small $x$ and undershoot them at larger $x$, cf. Fig. 5 .

The value of $\alpha_{s}$ is a necessary ingredient of all QCD calculations and it should be consistent with the PDFs employed. In our analysis we provide this consistency fitting $\alpha_{s}$ simultaneously with the other parameters of the data model. In this way we obtain the value of

$$
\alpha_{s}\left(M_{Z}\right)=0.1134 \pm 0.0011 \quad(\text { at NNLO) }
$$

and

$$
\alpha_{s}\left(M_{Z}\right)=0.1180 \pm 0.0012 \quad(\text { at NLO })
$$

with the uncertainties corresponding to the $68 \%$ C.L. They are calculated with the standard statistical criterion $\Delta \chi^{2}=1$ taking into account correlations of the systematic uncertainties in the data within the covariance matrix approach [50]. Both values in Eqs. $(3,4)$ are at variance due to the $O(0.005)$ scale variation error at NLO not included in the errors in Eqs. $(3,4)$. Once $\alpha_{s}$ is fitted simultaneously with the PDFs and the highertwist terms in the DIS SFs the errors in Eqs. $(3,4)$ take into account uncertainties in the latter as well. The ABM11 value of $\alpha_{s}$ is in a very good agreement with our earlier ABKM09 value and the error is somewhat smaller due to the improved accuracy of the HERA data. The profiles of $\chi^{2}$ versus $\alpha_{s}$, obtained in the NLO and NNLO variants of the ABM11 analysis with the value of $\alpha_{s}$ fixed and all other parameters fitted, are nearly parabolic at the minimum with the shapes determined by Eqs. $(3,4)$, cf. Fig. 6 . This gives an additional justifications of using the standard statistical criteria to estimate the parameter uncertainties in our fit. The $\chi^{2}$ profiles for the separate data sets employed in the fit are also nearly parabolic, cf. Fig. 7. The value of $\alpha_{s}$ preferred by the HERA and BCDMS data are in a good agreement, while the NMC and SLAC data prefer somewhat smaller and bigger value, respectively. The SLAC and the NMC data are sensitive to the higher-twist contribution, cf. discussion in $[18,54]$. Note, the cut on the 

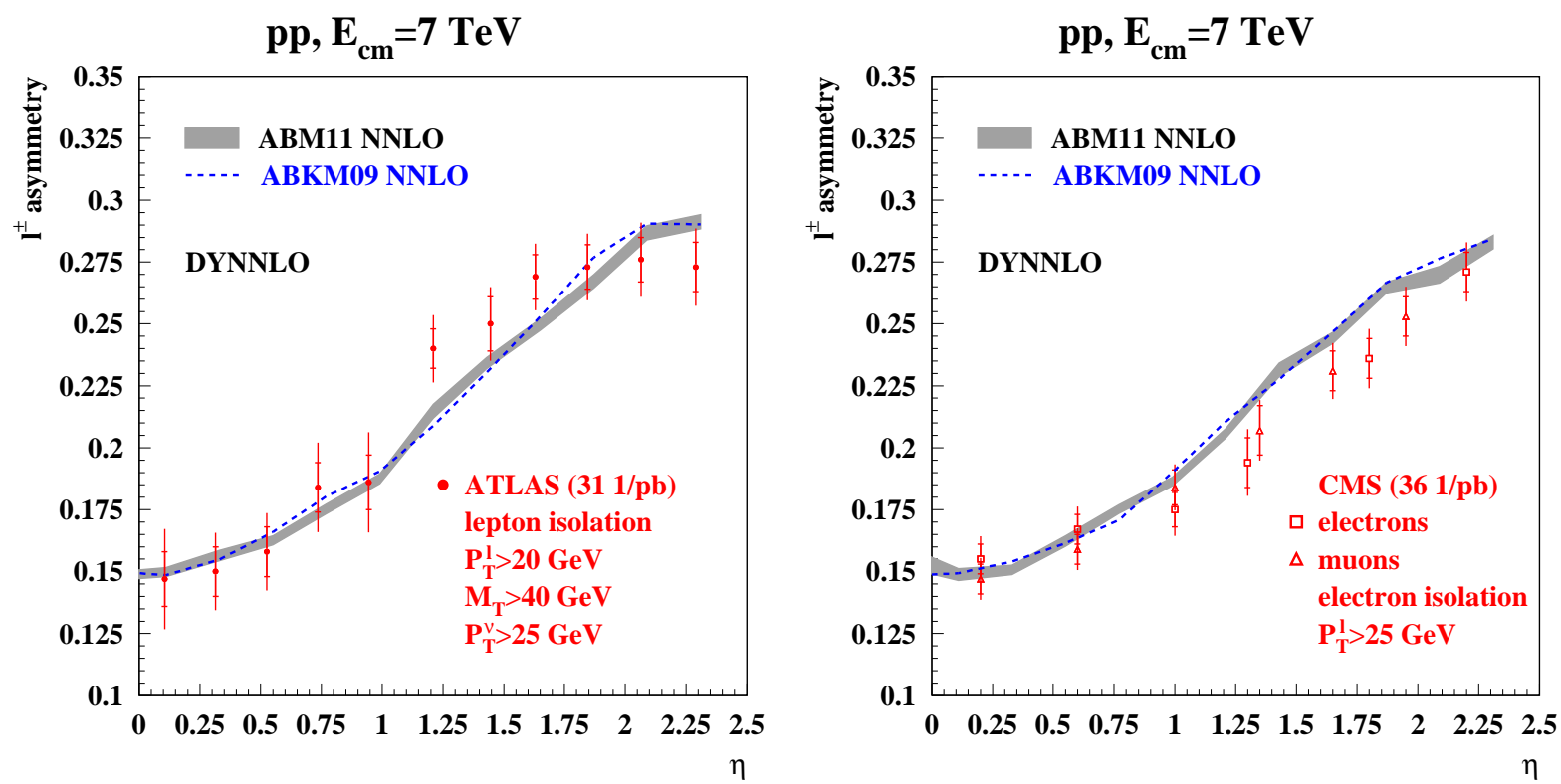

Figure 9: The data on charged-lepton asymmetry versus the lepton pseudorapidity $\eta$ obtained by the ATLAS [51] (left panel) and CMS [52] (right panel) experiments compared to the NNLO predictions based on the DYNNLO code of Ref. [53] and the ABM11 NNLO PDFs (shaded area showing the integration uncertainties). The ABKM09 NNLO predictions are given for comparison by dashes, without the integration uncertainties shown [18].

hadronic invariant mass $W$, which is commonly used in the global PDF fits, does not allow to get rid of the impact of the higher-twist terms on $\alpha_{s}$. Indeed, in a variant of our fit with the cut of $W^{2}>12.5 \mathrm{GeV}^{2}$ imposed and all higher-twist terms fixed at 0 we obtain $\alpha_{s}\left(M_{Z}\right)=$ $0.1191 \pm 0.0006$ at NNLO. This is substantially bigger than the value of $\alpha_{s}$ in Eq. (3). It is worth noting that in this way we approach the values of $\alpha_{s}$ obtained in the PDF fits [20, 55], performed with no higher-twist terms are taken into account. The value of $\alpha_{s}$ is also sensitive to the treatment of the correlated uncertainties in the data. E.g. in the NNLO MSTW fit [20] the HERA and NMC data prefer value of $\alpha_{s}\left(M_{Z}\right) \gtrsim 0.12$, contrary to our findings, cf. Fig. 7. Note that in [20] the NMC and HERA systematics errors are combined in quadrature. To study impact of this approximation we performed a trial NNLO ABKM09 fit with the same treatment of the NMC and HERA systematics and found that the value of $\alpha_{s}\left(M_{Z}\right)$ shifted up by +0.0029 as compared to the nominal ABKM value. Many other aspects of our analysis, which may affect the value of $\alpha_{s}$, are also different from $[20,55]$ : basic relations for the DIS cross sections, data normalization, etc, cf. Ref. [18] for a detailed discussion. These differences make a detailed comparison of our results with $[20,55]$ difficult.

While the 3-flavor FFN scheme is nicely sufficient for

\begin{tabular}{|l|c|c|}
\hline \multirow{2}{*}{ Experiment } & \multicolumn{2}{|c|}{$\alpha_{s}\left(M_{Z}\right)$} \\
\cline { 2 - 3 } & $\mathrm{NLO}$ & NNLO $^{*}$ \\
\hline D0 1 jet & $0.1190 \pm 0.0011$ & $0.1149(12)$ \\
D0 2 jet & $0.1174(9)$ & $0.1145(9)$ \\
CDF 1 jet (cone) & $0.1181(9) 9$ & $0.1134(9)$ \\
CDF 1 jet $\left(k_{\perp}\right)$ & $0.1181(10)$ & $0.1143(9)$ \\
\hline ABM11 & $0.1180(12)$ & $0.1134(11)$ \\
\hline
\end{tabular}

Table 1: The values of $\alpha_{s}\left(M_{Z}\right)$ based on including individual data sets of Tevatron jet data $[56,57,58,59]$ into the analysis at NLO. The NNLO* fit refers to the NNLO analysis of the DIS and DY data together with the NLO and soft gluon resummation corrections (next-to-leading logarithmic accuracy) for the 1 jet inclusive data, cf. $[60,61]$.

the description of the DIS data, the 5-flavor factorization schemes is most often justified for the collider phenomenology in view of much bigger scales involved. In cases the 4-flavor scheme may be also relevant. The 4(5)-flavor ABM11 PDFs are matched with the 3(4)flavor ones at the scale of $m_{c}$ and $m_{b}$, respectively, employing massive OMEs taken in the running-mass definition [9]. The 4- and 5-flavor PDFs at larger scales are generated by means of the massless QCD evolution with these boundary conditions. The heavy-quark PDFs are particularly sensitive to the values of $m_{c}, m_{b}$. Taking 


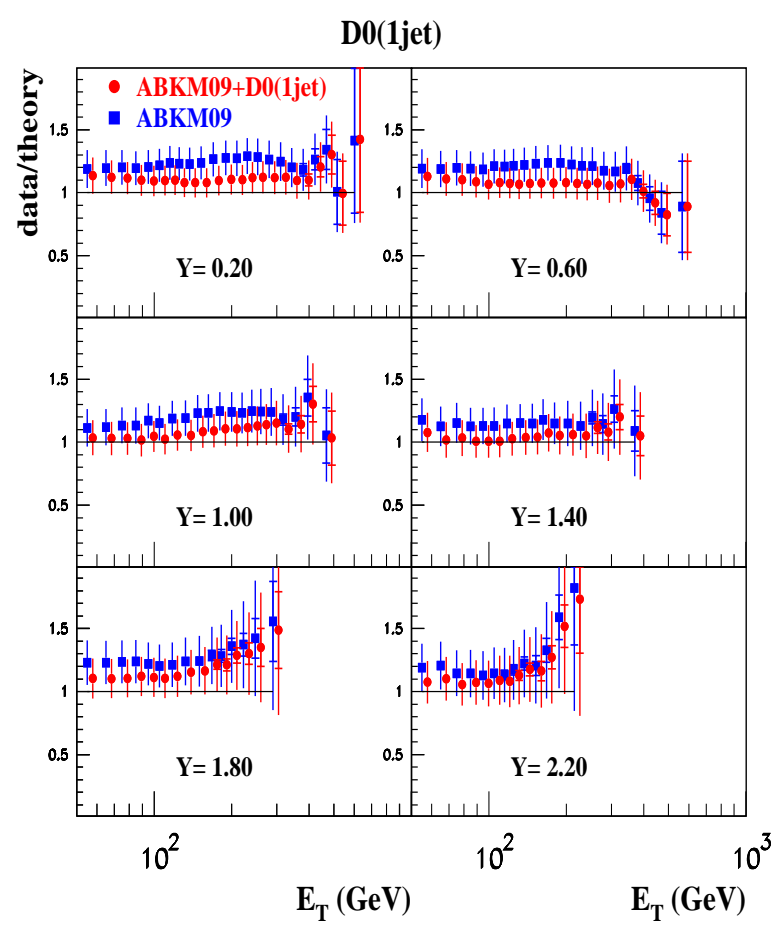

Figure 10: Cross section data for 1-jet inclusive production from the D0 collaboration [58] as a function of the jet's transverse energy $E_{T}$ for the renormalization and factorization scales equal to $E_{T}$ compared to the result of [12] (circles) and a re-fit including this data (squares) including the NNLO threshold re-summation corrections to the jet production [60].

advantage of the $\overline{\mathrm{MS}}$ definition we fix them at the PDG values of Eqs.(1,2). Due to the change in the heavy flavor treatment and impact of the new data included into the fit, the ABM11 heavy-quark PDFs differ from the ABKM09 ones, cf. Fig. 8. The uncertainties in the heavy-quark PDFs are to a large extend defined by the uncertainties in $m_{c}, m_{b}$. In our fit the latter are calculated as a sensitivity of the fitted data supplemented by the PDG constraints of Eqs. $(1,2)$ to $m_{c}, m_{b}$. The uncertainty in $m_{b}$ obtained in this way coincides with one in Eq.(1), while for $m_{c}$ it reduces to $0.06 \mathrm{GeV}$ due to impact of the inclusive HERA data. The uncertainties in the heavy-quark PDFs estimated with these constraints on $m_{c}, m_{b}$ are essentially reduced as compared to the ABKM09 case. This improvement is especially important for the hadronic single-top production driven by the initial-state $b$-quarks and for the Higgs production through the vector-boson-fusion channel, which is sensitive to the $c$-quark distribution [62].

The hadronic jet production provides an additional

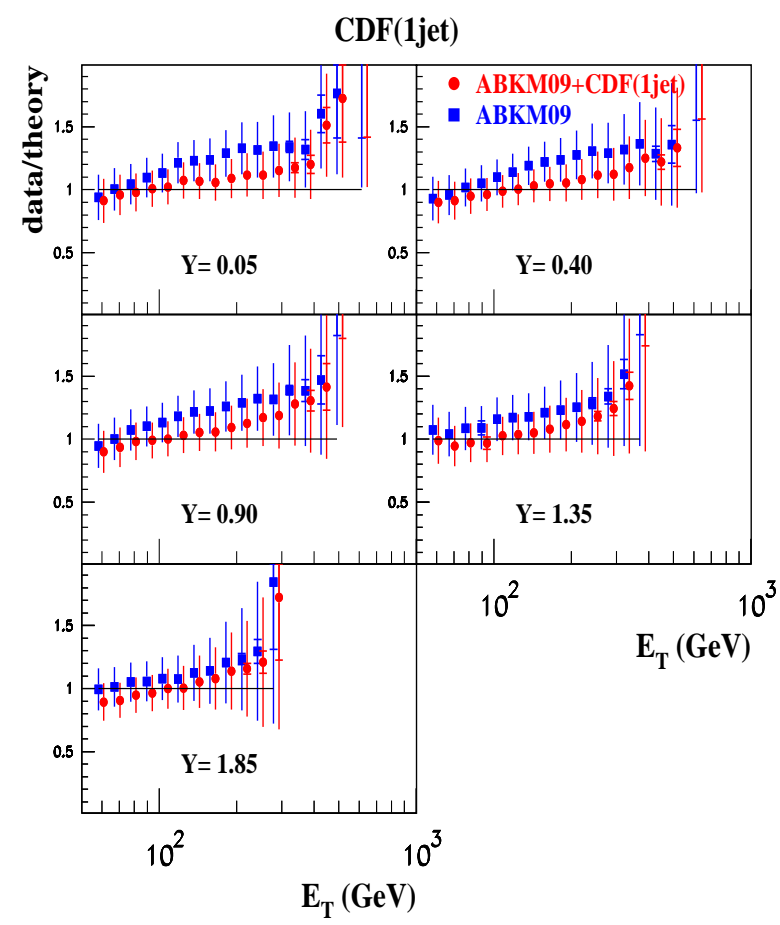

Figure 11: Same as Fig. 10 for the cross section data for 1-jet inclusive production from the CDF collaboration using a $k_{T}$ jet algorithm [56].

constraint on the PDFs, in particular on the large- $x$ gluon distribution $[20,39,19]$. However, the calculation of the full NNLO QCD corrections to this process is still in progress (see $[63,64]$ and references therein). This precludes a consistent use of the Tevatron jet data in our NNLO PDF fit. Nevertheless, in order to check any potential impact of the jet Tevatron data on our PDFs we have performed trial variants of the NNLO ABKM09 fit with the Tevatron jet data added [61]. The NLO QCD corrections [65, 66] and the partial (soft gluon enhanced) NNLO corrections due to threshold resummation [60] have been computed with the FastNLO tool $[67,68]$.

In general, the Tevatron jet data overshoot the ABKM09 predictions, nevertheless they can be smoothly accommodated in the fit. The typical value of $\chi^{2} / N D P \approx 1$ is achieved with account of the error correlations for the jet data sets of $[58,59,56,57]$ once they are included into the NNLO ABKM09 fit. Meanwhile the various data sets demonstrate a somewhat different trend with respect to the ABKM09 predictions. E.g., the off-set of the D0 inclusive jet data [58] does not depend on the jet energy $E_{T}$ and therefore may be attributed to 


\begin{tabular}{|c|c|c|c|c|c|}
\hline$\sigma(H)[p b]$ & ABKM09 & D0 1-jet inc. & D0 di-jet & $\begin{array}{c}\text { CDF 1-jet inc. } \\
(\text { cone })\end{array}$ & $\begin{array}{c}\text { CDF 1-jet inc. } \\
\left(k_{T}\right)\end{array}$ \\
\hline $\begin{array}{c}\text { Tevatron(1.96) } \\
\text { LHC(7) }\end{array}$ & $\mathbf{0 . 7 7 0 ( 5 0 )}$ & $0.859(29)$ & $0.833(27)$ & $0.815(25)$ & $0.842(25)$ \\
$\mathbf{1 4 . 3 4 ( 4 1 )}$ & $14.68(29)$ & $14.69(27)$ & $14.11(28)$ & $14.44(27)$ \\
\hline
\end{tabular}

Table 2: The predicted cross sections for Higgs boson production in ggF with $m_{H}=120 \mathrm{GeV}$ at Tevatron $(\sqrt{s}=1.96 \mathrm{TeV})$ and at LHC $(\sqrt{s}=7 \mathrm{TeV})$ from the NNLO variants of the ABKM09 fit [12] corresponding to Tab. 1. The uncertainty in brackets refers to the $1 \sigma$ standard deviation for the combined uncertainty on the PDFs and the value of $\alpha_{s}\left(M_{Z}\right)$. The values in bold correspond to the published result [69].

the impact of the currently missing full NNLO corrections, cf. Figs. 10 and 11. In contrast, for the CDF data of [56] obtained with the $k_{T}$ jet algorithm the pulls rise with $E_{T}$ and can be reduced only by means of a modification of the PDF shapes.

The values of $\alpha_{s}$ extracted from the trial ABKM09 fits with the Tevatron jet data included are compared with the nominal ABKM09 value in Tab. 1. At most, they are bigger by $1 \sigma$, while for the CDF cone jet algorithm data [57] the central value of $\alpha_{s}$ is even the same. The predictions for the light Higgs production cross section, which are defined by the gluon distribution at $x \lesssim 0.1$, are also not very sensitive to the constraints coming from the Tevatron data, cf. Tab. 2. The impact of the Tevatron jet data on the large- $x$ gluon distribution is more significant. However, in this context we note that the trend of the first LHC data on the jet production with respect to the various PDF predictions is different from the Tevatron measurements. The ABKM09 predictions are in better agreement with the CMS and ATLAS inclusive data of $[70,71]$ than the predictions based on the PDFs of $[20,39,19]$, which were tuned to the Tevatron inclusive jet data. Jet data from LHC is still subject to large systematic errors, though. Note also that the Tevatron dijet and 3-jet production data are in good agreement with the ABKM09 predictions [72], in contrast to the case of inclusive jet production at Tevatron. These ambiguities in the data as well as the limitations in the current theoretical treatment prevent the use of hadronic jet data in our fit.

The data on $W / Z$ productions being produced by the LHC experiments also may help to improve the PDF accuracy. The charged-lepton asymmetry data [51, 52] obtained by the ATLAS and CMS experiments are compared to the NNLO predictions based on the ABM11 PDFs in Fig. 9. All differential distributions for $W$ - and $Z$-boson production are computed with the fully exclusive NNLO program DYNNLO [53], which allows to take into account the kinematical cuts imposed in the experiments, cf. Fig. 9. The overall agreement with both experiments is sufficiently good. At values of $\eta \sim 1.5$ for the lepton pseudo-rapidity $\eta$ the data show a different trend with respect to the predictions, however the discrepancy is within the data uncertainties. Preliminary data on the charge-lepton asymmetry at large rapidity obtained by the $\mathrm{LHCb}$ collaboration [73] are also in good agreement with the ABKM09 predictions. To check the impact of the LHC charged-lepton asymmetry data on our fit, we have performed a variant of the ABM11 analysis which consists of adding the data of $[51,52]$ to the fit. We have found that the impact of those data is only marginal in view of still big experimental uncertainties.

Summary. We have discussed different schemes for the treatment of the heavy flavor component in DIS. In the kinematic region $Q^{2} \simeq m_{h}^{2}$ all schemes need to match to the 3-flavor scheme, which allows for a consistent comparison of the various PDF sets. We have performed this benchmark exercise with the help to the OPENQCDRAD code for standardized precision comparison. Within the framework of the ABM11 PDF analysis, which uses the running mass scheme for the heavy quarks, we find that the FFN is completely sufficient for describing the existing DIS data. We have also given detailed information on theoretical and experimental improvements which are of relevance for the low- $x$ PDFs, especially for the gluon, and we have briefly sketched the implications for LHC phenomenology, so that differences of ABM11 with respect to other PDF sets can be explained. More benchmark comparisons with the help of OPENQCDRAD can be found elsewhere [18] and are expected in the future.

Acknowledgments. This work has been supported by Helmholtz Gemeinschaft under contract VH-HA101 (Alliance Physics at the Terascale), by the Deutsche Forschungsgemeinschaft in Sonderforschungsbereich/Transregio 9 and by the European Commission through contract PITN-GA-2010-264564 (LHCPhenoNet). 


\section{References}

[1] E. Witten, Heavy Quark Contributions to Deep-Inelastic Scattering, Nucl. Phys. B104 (1976) 445-476. doi:10.1016/05503213(76)90111-5.

[2] E. Laenen, S. Riemersma, J. Smith, W. van Neerven, Complete $O\left(\alpha_{s}\right)$ corrections to heavy flavor structure functions in electroproduction, Nucl. Phys. B392 (1993) 162-228. doi:10.1016/0550-3213(93)90201-Y.

[3] T. Gottschalk, Chromodynamic corrections to neutrino production of heavy quarks, Phys. Rev. D23 (1981) 56. doi:10.1103/PhysRevD.23.56.

[4] M. Glück, S. Kretzer, E. Reya, The strange sea density and charm production in deep-inelastic charged current processes, Phys. Lett. B380 (1996) 171-176, [Erratum-ibid. B405, 391 (1997)]. arXiv:hep-ph/9603304, doi:10.1016/03702693(96)00456-X.

[5] E. Laenen, S.-O. Moch, Soft gluon resummation for heavy quark electroproduction, Phys. Rev. D59 (1999) 034027. arXiv:hep-ph/9809550, doi:10.1103/PhysRevD.59.034027.

[6] G. Corcella, A. D. Mitov, Soft gluon resummation for heavy quark production in charged current deep-inelastic scattering, Nucl. Phys. B676 (2004) 346-364. arXiv:hep-ph/0308105, doi:10.1016/j.nuclphysb.2003.10.027.

[7] S. Alekhin, S. Moch, Higher order QCD corrections to chargedlepton deep-inelastic scattering and global fits of parton distributions, Phys. Lett. B672 (2009) 166-171. arXiv:0811.1412.

[8] N. Lo Presti, H. Kawamura, S. Moch, A. Vogt, Thresholdimproved predictions for charm production in deep-inelastic scattering, PoS DIS2010 (2010) 163. arXiv:1008.0951.

[9] S. Alekhin, S. Moch, Heavy-quark deep-inelastic scattering with a running mass, Phys. Lett. B699 (2011) 345-353. arXiv:1011.5790, doi:10.1016/j.physletb.2011.04.026.

[10] F. Aaron, et al., Combined Measurement and QCD Analysis of the Inclusive $e^{ \pm} p$ Scattering Cross Sections at HERA, JHEP 1001 (2010) 109. arXiv:0911.0884, doi:10.1007/JHEP01(2010)109.

[11] K. Nakamura, et al., Review of particle physics, J. Phys. G37 (2010) 075021. doi:10.1088/0954-3899/37/7A/075021.

[12] S. Alekhin, J. Blümlein, S. Klein, S. Moch, The 3, 4, and 5-flavor NNLO Parton from Deep-Inelastic-Scattering Data and at Hadron Colliders, Phys. Rev. D81 (2010) 014032. arXiv:0908.2766, doi:10.1103/PhysRevD.81.014032.

[13] C. Adloff, et al., Deep-inelastic inclusive ep scattering at low $\mathrm{x}$ and a determination of $\alpha_{s}$, Eur. Phys. J. C21 (2001) 33-61. arXiv:hep-ex/0012053, doi:10.1007/s100520100720.

[14] S. Chekanov, et al., Measurement of the neutral current crosssection and $F_{2}$ structure function for deep-inelastic $e^{+} p$ scattering at HERA, Eur. Phys. J. C21 (2001) 443-471. arXiv:hepex/0105090, doi:10.1007/s100520100749.

[15] S. Alekhin, S. A. Kulagin, R. Petti, Determination of Strange Sea Distributions from Neutrino-Nucleon Deep-Inelastic Scattering, Phys. Lett. B675 (2009) 433-440. arXiv:0812.4448, doi:10.1016/j.physletb.2009.04.033.

[16] D. Boer, M. Diehl, R. Milner, R. Venugopalan, W. Vogelsang, et al., Gluons and the quark sea at high energies: distributions, polarization, tomography, arXiv:1108.1713.

[17] F. Aaron, C. Alexa, V. Andreev, S. Backovic, A. Baghdasaryan, et al., Measurement of the Inclusive $e^{ \pm} p$ Scattering Cross Section at High Inelasticity y and of the Structure Function $F_{L}$, Eur. Phys. J. C71 (2011) 1579. arXiv:1012.4355, doi:10.1140/epjc/s10052-011-1579-4.

[18] S. Alekhin, J. Blümlein, S. Moch, Parton distribution functions and benchmark cross sections at NNLO, arXiv:1202.2281.

[19] R. D. Ball, V. Bertone, F. Cerutti, L. Del Debbio, S. Forte, et al., Impact of Heavy Quark Masses on Parton Distributions and LHC Phenomenology, Nucl. Phys. B849 (2011) 296-363. arXiv:1101.1300, doi:10.1016/j.nuclphysb.2011.03.021.

[20] A. Martin, W. Stirling, R. Thorne, G. Watt, Parton distributions for the LHC, Eur. Phys. J. C63 (2009) 189-285. arXiv:0901.0002, doi:10.1140/epjc/s10052-009-1072-5.

[21] P. Jimenez-Delgado, E. Reya, Dynamical NNLO parton distributions, Phys. Rev. D79 (2009) 074023. arXiv:0810.4274, doi:10.1103/PhysRevD.79.074023.

[22] F. Aaron, et al., Measurement of $D^{ \pm}$Meson Production and Determination of $F_{2}^{c \bar{c}}$ at low $Q^{2}$ in Deep-Inelastic Scattering at HERA, Eur. Phys. J. C71 (2011) 1769. arXiv:1106.1028, doi:10.1140/epjc/s10052-011-1769-0.

[23] B. Harris, J. Smith, Heavy quark correlations in deepinelastic electroproduction, Nucl. Phys. B452 (1995) 109-160. arXiv:hep-ph/9503484, doi:10.1016/0550-3213(95)00256-R.

[24] R. D. Ball, et al., Unbiased global determination of parton distributions and their uncertainties at NNLO and at LO, Nucl. Phys. B855 (2012) 153-221. arXiv:1107.2652, doi:10.1016/j.nuclphysb.2011.09.024.

[25] M. Arneodo, et al., Measurement of the proton and deuteron structure functions, $F_{2}(\mathrm{p})$ and $F_{2}(\mathrm{~d})$, and of the ratio $\sigma_{L} / \sigma_{T}$, Nucl. Phys. B483 (1997) 3-43. arXiv:hep-ph/9610231, doi:10.1016/S0550-3213(96)00538-X.

[26] A. Benvenuti, et al., A High Statistics Measurement of the Proton Structure Functions $F_{2}\left(x, Q^{2}\right)$ and $R$ from Deep-Inelastic Muon Scattering at High $Q^{2}$, Phys. Lett. B223 (1989) 485. doi:10.1016/0370-2693(89)91637-7.

[27] A. Benvenuti, et al., A high statistics measurement of the deuteron structure functions $F_{2}\left(x, Q^{2}\right)$ and $\mathrm{R}$ from deepinelastic muon scattering at high $Q^{2}$, Phys. Lett. B237 (1990) 592. doi:10.1016/0370-2693(90)91231-Y.

[28] L. Whitlow, S. Rock, A. Bodek, E. Riordan, S. Dasu, A Precise extraction of $R=\sigma_{L} / \sigma_{T}$ from a global analysis of the SLAC deep-inelastic ep and ed scattering cross-sections, Phys. Lett. B250 (1990) 193-198. doi:10.1016/0370-2693(90)91176-C.

[29] A. Bodek, M. Breidenbach, D. Dubin, J. Elias, J. I. Friedman, et al., Experimental Studies of the Neutron and Proton Electromagnetic Structure Functions, Phys. Rev. D20 (1979) 14711552. doi:10.1103/PhysRevD.20.1471.

[30] W. Atwood, E. D. Bloom, R. Cottrell, H. DeStaebler, M. Mestayer, et al., Inelastic electron Scattering from Hydrogen at 50-Degrees and 60-Degrees, Phys. Lett. B64 (1976) 479. doi:10.1016/0370-2693(76)90127-1.

[31] M. Mestayer, W. Atwood, E. D. Bloom, R. Cottrell, H. DeStaebler, et al., The ratio $\sigma_{L} / \sigma_{T}$ from deep-inelastic electron scattering, Phys. Rev. D27 (1983) 285. doi:10.1103/PhysRevD.27.285.

[32] J. Gomez, R. Arnold, P. E. Bosted, C. Chang, A. Katramatou, et al., Measurement of the A-dependence of deepinelastic electron scattering, Phys. Rev. D49 (1994) 4348-4372. doi:10.1103/PhysRevD.49.4348.

[33] S. Dasu, P. deBarbaro, A. Bodek, H. Harada, M. Krasny, et al., Measurement of kinematic and nuclear dependence of $R=$ $\sigma_{L} / \sigma_{T}$ in deep-inelastic electron scattering, Phys. Rev. D49 (1994) 5641-5670. doi:10.1103/PhysRevD.49.5641.

[34] M. Virchaux, A. Milsztajn, A Measurement of $\alpha_{s}$ and higher twists from a QCD analysis of high statistics $F_{2}$ data on hydrogen and deuterium targets, Phys. Lett. B274 (1992) 221-229. doi:10.1016/0370-2693(92)90527-B.

[35] S. A. Kulagin, R. Petti, Global study of nuclear structure functions, Nucl. Phys. A765 (2006) 126-187. arXiv:hepph/0412425, doi:10.1016/j.nuclphysa.2005.10.011.

[36] M. Glück, E. Reya, M. Stratmann, Heavy quarks at high-energy colliders, Nucl. Phys. B422 (1994) 37-56. doi:10.1016/05503213(94)00131-6. 
[37] J. Vermaseren, A. Vogt, S. Moch, The Third-order QCD corrections to deep-inelastic scattering by photon exchange, Nucl. Phys. B724 (2005) 3-182. arXiv:hep-ph/0504242, doi:10.1016/j.nuclphysb.2005.06.020.

[38] F. Caola, S. Forte, J. Rojo, Deviations from NLO QCD evolution in inclusive HERA data, Phys. Lett. B686 (2010) 127-135. arXiv:0910.3143, doi:10.1016/j.physletb.2010.02.043.

[39] H.-L. Lai, M. Guzzi, J. Huston, Z. Li, P. M. Nadolsky, et al., New parton distributions for collider physics, Phys. Rev. D82 (2010) 074024. arXiv:1007.2241, doi:10.1103/PhysRevD.82.074024.

[40] M. Buza, Y. Matiounine, J. Smith, W. van Neerven, Charm electroproduction viewed in the variable flavor number scheme versus fixed order perturbation theory, Eur. Phys. J. C1 (1998) 301320. arXiv:hep-ph/9612398.

[41] I. Bierenbaum, J. Blümlein, S. Klein, The Gluonic Operator Matrix Elements at $O\left(\alpha_{s}^{2}\right)$ for DIS Heavy Flavor Production, Phys. Lett. B672 (2009) 401-406. arXiv:0901.0669, doi:10.1016/j.physletb.2009.01.057.

[42] I. Bierenbaum, J. Blümlein, S. Klein, Mellin Moments of the $O\left(\alpha_{s}^{3}\right)$ Heavy Flavor Contributions to unpolarized DeepInelastic Scattering at $Q^{2} \gg m^{2}$ and Anomalous Dimensions, Nucl. Phys. B820 (2009) 417-482. arXiv:0904.3563, doi:10.1016/j.nuclphysb.2009.06.005.

[43] J. Ablinger, J. Blümlein, S. Klein, C. Schneider, F. Wissbrock, The $O\left(\alpha_{s}^{3}\right)$ Massive Operator Matrix Elements of $O\left(n_{f}\right)$ for the Structure Function $F_{2}\left(x, Q^{2}\right)$ and Transversity, Nucl. Phys. B844 (2011) 26-54. arXiv:1008.3347, doi:10.1016/j.nuclphysb.2010.10.021.

[44] M. Aivazis, J. C. Collins, F. I. Olness, W.-K. Tung, Leptoproduction of heavy quarks. 2. A Unified QCD formulation of charged and neutral current processes from fixed target to collider energies, Phys. Rev. D50 (1994) 3102-3118. arXiv:hepph/9312319, doi:10.1103/PhysRevD.50.3102.

[45] R. Thorne, The Effect of Changes of Variable Flavour Number Scheme on PDFs and Predicted Cross Sections, arXiv:1201.6180.

[46] M. Guzzi, P. M. Nadolsky, H.-L. Lai, C.-P. Yuan, Generalmass treatment for deep-inelastic scattering at two-loop accuracy, arXiv: 1108.5112.

[47] A. Chuvakin, J. Smith, W. van Neerven, Comparison between variable flavor number schemes for charm quark electroproduction, Phys. Rev. D61 (2000) 096004. arXiv:hep-ph/9910250, doi:10.1103/PhysRevD.61.096004.

[48] K. Lipka, these proceedings.

[49] S. Forte, E. Laenen, P. Nason, J. Rojo, Heavy quarks in deep-inelastic scattering, Nucl. Phys. B834 (2010) 116-162. arXiv:1001.2312, doi:10.1016/j.nuclphysb.2010.03.014.

[50] S. I. Alekhin, Statistical properties of the estimator using covariance matrix, arXiv:hep-ex/0005042.

[51] G. Aad, et al., Measurement of the Muon Charge Asymmetry from $\mathrm{W}$ Bosons Produced in $p p$ Collisions at $\sqrt{s}=7 \mathrm{TeV}$ with the ATLAS detector, Phys. Lett. B701 (2011) 31-49. arXiv:1103.2929, doi:10.1016/j.physletb.2011.05.024.

[52] S. Chatrchyan, et al., Measurement of the lepton charge asymmetry in inclusive $W$ production in pp collisions at $\sqrt{s}=7 \mathrm{TeV}$, JHEP 1104 (2011) 050. arXiv:1103.3470, doi:10.1007/JHEP04(2011)050.

[53] S. Catani, G. Ferrera, M. Grazzini, W boson production at hadron colliders: the lepton charge asymmetry in NNLO QCD, JHEP 1005 (2010) 006. arXiv:1002.3115, doi:10.1007/JHEP05(2010)006.

[54] S. Alekhin, J. Blümlein, S. Moch, Higher order constraints on the Higgs production rate from fixed-target DIS data, Eur. Phys. J. C71 (2011) 1723. arXiv:1101.5261, doi:10.1140/epjc/s10052-011-1723-1.

[55] R. D. Ball, V. Bertone, L. Del Debbio, S. Forte, A. Guffanti, et al., Precision NNLO determination of $\alpha_{s}\left(M_{Z}\right)$ using an unbiased global parton set, Phys. Lett. B707 (2012) 66-71. arXiv:1110.2483, doi:10.1016/j.physletb.2011.11.053.

[56] A. Abulencia, et al., Measurement of the inclusive jet cross section using the $k_{\mathrm{T}}$ algorithm in $p \bar{p}$ collisions at $\sqrt{s}=1.96 \mathrm{TeV}$ with the CDF II detector, Phys. Rev. D75 (2007) 092006. arXiv:hep-ex/0701051, doi:10.1103/PhysRevD.75.092006, 10.1103/PhysRevD.75.119901.

[57] T. Aaltonen, et al., Measurement of the inclusive jet cross section at the Fermilab Tevatron $p \bar{p}$ collider using a cone-based jet algorithm, Phys. Rev. D78 (2008) 052006. arXiv:0807.2204, doi:10.1103/PhysRevD.78.052006, 10.1103/PhysRevD.79.119902.

[58] V. Abazov, et al., Measurement of the inclusive jet cross-section in $p \bar{p}$ collisions at $\sqrt{s}=1.96 \mathrm{TeV}$, Phys. Rev. Lett. 101 (2008) 062001. arXiv:0802.2400, doi:10.1103/PhysRevLett.101.062001.

[59] V. Abazov, et al., Measurement of the dijet invariant mass cross section in proton anti-proton collisions at $\sqrt{\mathrm{s}}=$ 1.96 TeV, Phys. Lett. B693 (2010) 531-538. arXiv:1002.4594, doi:10.1016/j.physletb.2010.09.013.

[60] N. Kidonakis, J. Owens, Effects of higher order threshold corrections in high $\mathrm{E}(\mathrm{T})$ jet production, Phys. Rev. D63 (2001) 054019. arXiv:hep-ph/0007268, doi:10.1103/PhysRevD.63.054019.

[61] S. Alekhin, J. Blümlein, S. Moch, Parton distributions and Tevatron jet data, arXiv:1105.5349.

[62] P. Bolzoni, F. Maltoni, S. Moch, M. Zaro, Higgs production via vector-boson fusion at NNLO in QCD, Phys. Rev. Lett. 105 (2010) 011801. arXiv:1003.4451.

[63] A. Gehrmann-De Ridder, E. Glover, J. Pires, Real-Virtual corrections for gluon scattering at NNLO, arXiv:1112.3613.

[64] P. Bolzoni, G. Somogyi, Z. Trocsanyi, A subtraction scheme for computing QCD jet cross sections at NNLO: integrating the iterated singly-unresolved subtraction terms, JHEP 1101 (2011) 059. arXiv:1011.1909, doi:10.1007/JHEP01(2011)059.

[65] Z. Nagy, Three jet cross-sections in hadron hadron collisions at next-to-leading order, Phys. Rev. Lett. 88 (2002) 122003. arXiv:hep-ph/0110315, doi:10.1103/PhysRevLett.88.122003.

[66] Z. Nagy, Next-to-leading order calculation of three jet observables in hadron hadron collision, Phys. Rev. D68 (2003) 094002. arXiv:hep-ph/0307268, doi:10.1103/PhysRevD.68.094002.

[67] T. Kluge, K. Rabbertz, M. Wobisch, FastNLO: Fast pQCD calculations for PDF fits, arXiv:hep-ph/0609285.

[68] M. Wobisch, D. Britzger, T. Kluge, K. Rabbertz, F. Stober, Theory-Data Comparisons for Jet Measurements in HadronInduced Processes, arXiv:1109.1310.

[69] S. Alekhin, J. Blümlein, P. Jimenez-Delgado, S. Moch, E. Reya, NNLO Benchmarks for Gauge and Higgs Boson Production at TeV Hadron Colliders, Phys. Lett. B697 (2011) 127-135. arXiv:1011.6259, doi:10.1016/j.physletb.2011.01.034.

[70] K. Rabbertz, Comparison of inclusive jet and dijet mass cross sections at $\sqrt{s}=7 \mathrm{TeV}$ with predictions of perturbative QCD, Tech. Rep. CMS-NOTE-2011-004, CERN, Geneva (Jun 2011).

[71] G. Aad, et al., Measurement of inclusive jet and dijet production in $p p$ collisions at $\sqrt{s}=7 \mathrm{TeV}$ using the ATLAS detector, arXiv:1112.6297.

[72] M. Wobisch, Recent QCD results from the Tevatron, arXiv: 1202.0205.

[73] Y. Amhis, Electroweak results at $\mathrm{LHCb}$, Presented at the 2011 Hadron Collider Physics symposium (HCP-2011), Paris, France, November 14-18 2011, 4 pages, 8 figures. arXiv:1202.0654. 